\title{
Impact of Althaea Officinalis extract in patients with atopic eczema: a double- blind randomized controlled trial
}

\author{
Negar Khalighi ${ }^{1}$, Farahzad Jabbari-Azad ${ }^{1}$, Maral Barzegar-Amini', Jalil Tavakkol-Afshari ${ }^{2}$, Pouran Layegh ${ }^{3}$ and \\ Roshanak Salari ${ }^{4^{*}}$ (D)
}

\begin{abstract}
Background: Althaea Officinalis introduced as an emerging anti-inflammatory agent with immunomodulatory effects that may suppress the symptoms of atopic eczema. In this study, we aimed to investigate the impact of liposomal formulation of Althaea Officinalis flower extract compared with steroids for atopic eczema treatment.

Methods: In this randomized, double-blind controlled trial phase II, forty patients with moderate to severe allergic atopic eczema confirmed by skin prick test were enrolled in the study from October 2018 to January 2019. Steroids on the lesions of the left side of the body and topical Althaea Officinalis extract (intervention) or eucerin as a topical placebo on the lesions of the right side of the body were tested. SCORAD (SCORing Atopic Dermatitis) was assessed at the baseline, and two, and four weeks after completing the trial. Data were analyzed by SPSS version 22.

Results: In the active treatment group, after two weeks, the SCORAD was significantly lower in the left side (recovery with steroid treatment) $(p<0.001)$, and after 4 weeks the SCORAD was lower on both sides, and there were no significant differences between two sides (similarity between Althaea Officinalis and steroid effects). In the control group, the SCORAD score was significantly lower on the left side (steroid) after two and four weeks ( $p<$ 0.001). There was no side effect and reaction to drugs.
\end{abstract}

Conclusions: Considering the potential side effects of steroids, and the similar efficacy of Althaea Officinalis flower extract, using this component may be accompanied by higher benefits in patients with atopic eczema.

Trial registration: IRCT2016102323235N5.

Keywords: Althaea Officinalis extract, Atopic eczema, SCORing Atopic Dermatitis

\section{Introduction}

Atopic eczema is one of the most prevalent diseases worldwide with a wide age distribution that is a debilitating medical condition affecting psychological and somatic functions $[1,2]$. Current data support the use of systemic therapies such as novel monoclonal antibodies and Janus kinase inhibitors beside systemic corticosteroids and

\footnotetext{
* Correspondence: SalariR@mums.ac.ir

${ }^{4}$ Department of Pharmaceutical Sciences in Persian Medicine, School of Persian and Complementary Medicine, Mashhad University of Medical Sciences, Mashhad, Iran

Full list of author information is available at the end of the article
}

antidepressants and topical therapies and topical treatments such as topical corticosteroids, antibiotics, and different eucerin in the treatment of atopic eczema with various severity indices [3]. Recently, alternative medicine using traditional herbal medicines has gained great interest, especially in refractory and severe cases in different age groups with or without the supervision of clinicians $[4,5]$. Components such as honey, beeswax, olive oil, and marshmallow (Althaea Officinalis) showed promising results on dermatologic disorders with autoimmune and/or inflammatory basis [6-8]. 
Althaea officinalis, (common name: Marshmallow), which showed in Fig. 1, is a perennial species indigenous to Europe, Western Asia, and North Africa, which is used in alternative medicine and as an ornamental plant [9]. Its active compounds modulate the immune system through stimulation of monocytes leading to secretion of interleukin-6 and tumor necrosis factor (TNF) $[10,11]$. Althaea Officinalis introduced as an emerging antiinflammatory agent [12] with immunomodulatory effects that may suppress the symptoms of atopic eczema. Therefore, it might suppress the symptoms of atopic eczema.

The prevalence of atopic dermatitis is increasing with the industrialization of communities. Regarding the effects of severe pruritus in patients, which is especially children, its mental health problems on the patients and parents, the side effects of topical steroids in high consumption and increasing the systemic absorption, the resistance and fear of parents in using topical steroids, and vice versa overuse and its side effects, we decided to find a suitable and low-risk solution to control the disease and itching. Due to the anti-inflammatory and itching effects of marshmallows, we aimed to investigate the efficacy and safety of topical liposomal formulation of Althaea officinalis flower extract for treating atopic eczema.

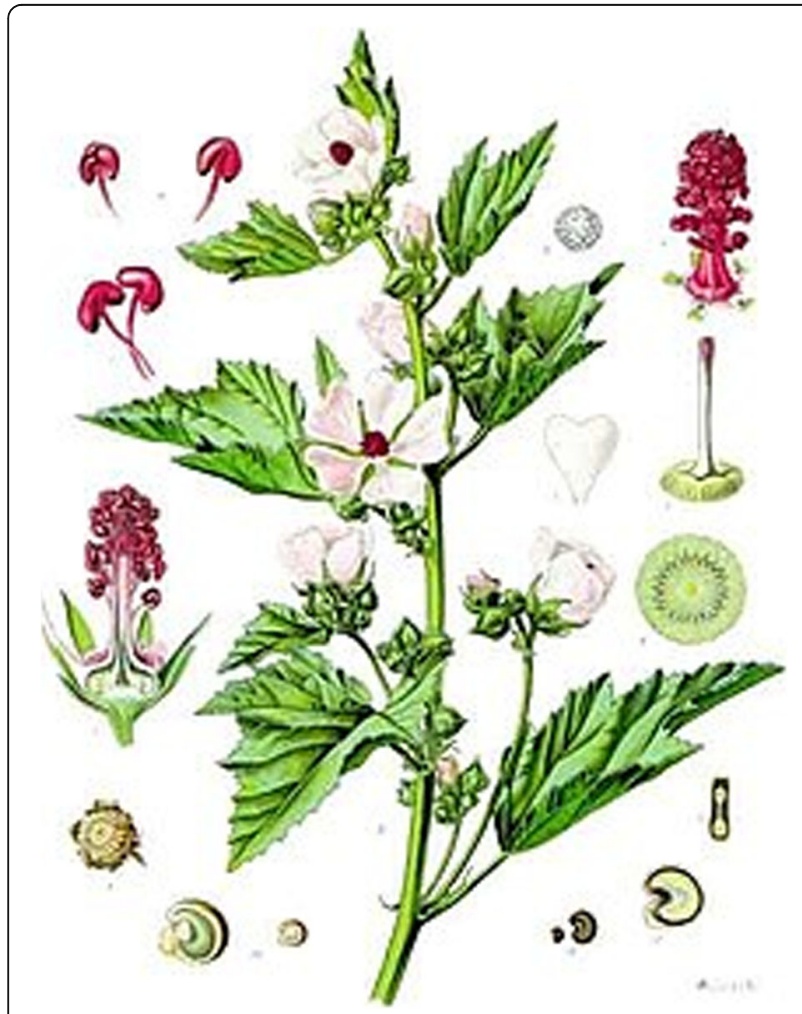

Fig. 1 Althaea Officinalis plant [13]

\section{Methods}

This was a randomized, double-blind controlled trial phase II in forty patients with atopic eczema. The trial was conducted from October 2018 to January 2019, at Qaem Educational Hospital; the largest tertiary referral hospital in Mashhad, Iran. This study is a pilot which considering of the other similar articles, 20 people in each group were selected and each patient had both intervention and control (two Body side selected) to prevent sensory effects (severity of itching that depends on the individual).

The active treatment group included 20 patients with moderate to severe allergic atopic eczema confirmed by skin prick test were received steroids (Hydrocortisone acetate ointment (1\%) or Triamcinolone, depending on the age of the patient) on the lesions of the left side of the body and topical Althaea officinalis extract (intervention) of the right side of their body.

In the control group, 20 patients with moderate to severe allergic atopic eczema confirmed by skin prick test were received steroids on the lesions of the left side of the body, and eucerin as a topical placebo on lesions of the right side of their body. To make all patients uniform and occurs no mistakes in completing the questionnaire, the left side was chosen for steroids (constant side).

Patients with simultaneous dermatologic disorders including bacterial infection, the patient using systemic immunosuppressive drugs, and patients who missed follow-up sessions showing poor compliance of them, were excluded. Also, before the randomization, all patients were tested for hyper-reactive responses and were excluded if they had them. The online random generator was used to generate random choices (https://www. random.org/).

The Intervention group received topical formulation of Althaea officinalis $10 \%$. In the summer of 2018, we bought the herb from the validated centers of medicinal herbs in Mashhad. The herbal extract was encapsulated in liposomes to improve its penetration through the skin. The active control group received topical steroids with a similar prescription pattern.

For the effect of these compounds and their comparison with steroids, it is necessary to remove confounding factors such as lesion severity, patient tolerance, skin thickness, and nutrition, etc. therefore, to achieve this goal, the effects of both drugs (marshmallow and hydrocortisone) were examined in each person. This means that both groups were prescribed topical steroids.

Patients or parents were asked to use the new formulation in half of the body (right) and steroids ointment in the second half (left). The duration of treatment was one month. Comparison of the Althaea Officinalis flower extracts liposomal formulation with steroids 
Table 1 Demographic data between two groups of case and control

\begin{tabular}{lllll}
\hline Variable & & Active treatment group & Active control group & P-value \\
\hline Subjects enrolled & & 20 & 20 & - \\
Sex & Male & $18(45 \%)$ & $16(40 \%)$ & 0.651 \\
& Female & $22(55 \%)$ & $24(60 \%)$ & $31.00(16.50-72.00)$ \\
Age (months) & & $34.00(15.75-90.00)$ & 0.787 \\
\hline
\end{tabular}

ointment in the relieving symptoms (itching, redness, bumps, scaling, and fissures) were done every 2 weeks (twice) after treatment by the therapist with patient history and the SCORAD questionnaire.

\section{Herbal medicine formulation}

In the summer of 2018, we bought the herb from the validated centers of medicinal herbs in Mashhad. To produce topical liposomal formulation of Althaea Officinalis, first, the hydroalcoholic extract of the Althaea Officinalis flowers were obtained by maceration method, in which the dried flowers were powdered then macerated in hydroalcoholic solvent ( $70 \%$ ethanol, $30 \%$ water) for $72 \mathrm{~h}$. After removing the solvent, the extract was collected.

Then, the extract is encapsulated in liposomes. To make a $20 \mathrm{cc}$ liposomal solution, $200 \mathrm{mg}$ cholesterol and $600 \mathrm{mg}$ lecithin were dissolved in chloroform. After the solvent was removed, the extract suspension in water was added to the bottom lipid layers of the balloon and the resulting suspension was homogenized. This suspension was then added to the topical base of Eucerin. The percentage of extract in this product was $10 \%$. The acute median lethal dose (LD50) of A. officinalis in mice was greater than $5,000 \mathrm{mg} / \mathrm{kg}$ [14], so the amount of extract that each patient used (less than $3 \mathrm{~g}$ ) is very less than the LD50, besides the patient used this product topically not orally.

\section{SCORing Atopic Dermatitis}

SCORAD (SCORing Atopic Dermatitis) was assessed at the baseline, and two and four weeks after completing the trial. All patients were evaluated for pruritus, redness, bulge, scaling, and fissure. The objective SCORAD is a scoring system based on the assessment of extent and intensity in a standardized manner. The extent of lesions is scored by applying the rule of nine after drawing the lesions on an evaluation form. The intensity is determined by grading the 6 items on a scale from 0 to 3 (erythema, edema/population, oozing/crusts, excoriation, lichenification, and dryness).

Data were analyzed by SPSS 25 using chi-square and independent-sample t-tests at the level of 0.05 .

\section{Ethics approval and consent to participate}

The protocol of the study was approved by the ethics committee of Mashhad University of Medical Sciences (code: IR.MUMS.fm.REC.1394.543). Written informed consent and verbal assent were obtained from patients involved before enrolment when data were collected.

\section{Results}

Twenty patients with atopic eczema in each group completed the trial. In this study, 34 (42.5\%) of all subjects were male (18 and 16 patients in treatment and control groups, respectively) and $46(57.5 \%)$ of them were female (22 and 24 patients in treatment and control groups, respectively). The distribution of age was nonparametric which in treatment and control group was 34 (15.75 -90) and 31 (16.50-72), respectively. According to the Table 1, both groups were similar in terms of age and distribution of gender.

The results of Table 2 shows mean SCORAD in active treatment group. Before the study, for both groups severity of atopic eczema in terms of SCORAD score had been determined, which was similar in the right and left

Table 2 Mean scoring of Atopic Dermatitis (SCORAD) in the active treatment group

\begin{tabular}{|c|c|c|c|}
\hline \multirow[t]{3}{*}{ Time } & \multicolumn{2}{|l|}{ Scores (Mean \pm SD) } & \multirow{3}{*}{$\begin{array}{l}\text { Between } \\
\text { right and } \\
\text { left half- } \\
\text { body } P \text { - } \\
\text { value }\end{array}$} \\
\hline & Right half-body & Left half-body & \\
\hline & Althaea Officinalis & Hydrocortisone acetate ointment (1\%) or Triamcinolone & \\
\hline 0 (baseline) & $14.55 \pm 2.21$ & $14.55 \pm 2.21$ & - \\
\hline Week 2 & $9.55 \pm 2.19$ & $5.90 \pm 1.97$ & $<0.001^{* *}$ \\
\hline$P$-value weeks $0-2$ & $<0.001^{* *}$ & $<0.001^{* *}$ & \\
\hline Week 4 & $1.00 \pm 1.34$ & $1.05 \pm 1.19$ & 0.716 \\
\hline$P$-value weeks $2-4$ & $<0.001^{* *}$ & $<0.001^{* *}$ & \\
\hline$P$-value weeks $0-4$ & $<0.001^{* *}$ & $<0.001^{* *}$ & \\
\hline
\end{tabular}


Table 3 Mean scoring of Atopic Dermatitis (SCORAD) in the active control group

\begin{tabular}{|c|c|c|c|}
\hline \multirow[t]{3}{*}{ Time } & \multicolumn{2}{|c|}{ Scores (Mean \pm SD) } & \multirow{3}{*}{$\begin{array}{l}\text { Between } \\
\text { right and } \\
\text { left half- } \\
\text { body } P \text { - } \\
\text { value }\end{array}$} \\
\hline & Right half-body & Left half-body & \\
\hline & Placebo & Hydrocortisone acetate ointment (1\%) or Triamcinolone & \\
\hline 0 (baseline) & $14.40 \pm 1.90$ & $14.40 \pm 1.90$ & - \\
\hline Week 2 & $13.05 \pm 2.04$ & $6.05 \pm 2.31$ & $<0.001$ \\
\hline$P$-value weeks $0-2$ & 0.287 & $<0.001$ & \\
\hline Week 4 & $11.75 \pm 1.94$ & $1.10 \pm 0.85$ & $<0.001$ \\
\hline$P$-value weeks $2-4$ & 0.003 & $<0.001$ & \\
\hline$P$-value weeks $0-4$ & $<0.001$ & $<0.001$ & \\
\hline
\end{tabular}

side in each patient. After that, SCORAD had been estimated in each group for left and right lesions every two weeks. In the active treatment group, after two weeks, the SCORAD was significantly lower on the left side (recovery with steroid treatment $)(P<0.001)$, and after four weeks the SCORAD was lower on both sides and there were no significant differences between the two sides $(P$ value $=0.716$ ). This results shows that Althaea Officinalis decreased SCORAD as well as topical triamcinolone after 4 weeks.

The results of Table 3 shows mean SCORAD in active control group. In the control group, the SCORAD score was significantly lower on the left side (steroid) than right side (placebo) after two and four weeks $(P<0.001)$. This results indicate that topical triamcinolone had a better effects in decreasing SCORAD than placebo. There was no side effect and reaction to drugs.

Figure 2 shows improvement from baseline in mean scoring of Atopic Dermatitis (SCORAD) Althaea Officinalis compared with Hydrocortisone acetate ointment (1\%) or Triamcinolone. Accordingly, topical triamcinolone had a better results than Althaea Officinalis in two weeks but the final result after 4 weeks was same.

\section{Discussion}

Our study showed that topical liposomal formulation of Althaea Officinalis flower extract had a similar effect to

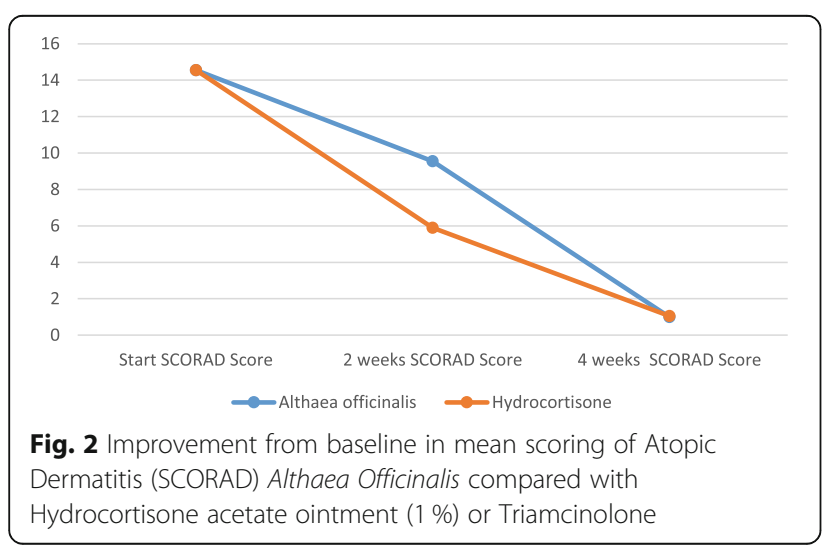

hydrocortisone acetate ointment (1\%) or topical triamcinolone in reduction of symptoms of patients suffering from atopic eczema with no remarkable side effects. Previous studies support the use of alternative medicine in the treatment of allergic skin conditions.

Hon et al. studies the role of herbal medicine for the treatment of atopic dermatitis and showed that prescription of traditional Chinese herbal medicine in these patients with atopic dermatitis improves the quality of life of patients and decreases the dose of corticosteroids [15]. Al-Waili used topical honey, beeswax, and olive oil mixture for treatment of atopic dermatitis or psoriasis and they reported its clinical efficacy [6]. In a review by Thandar et al., the topical herbal medicines for atopic eczema were reviewed, they found that current evidence is insufficient to make a general recommendation for using the herbal medicine in the treatment of allergic skin conditions [16].

Previous studies showed that Althaea Officinalis has several effects on the human body including its immunomodulatory effects by inhibiting the cytokine and interferon production and anti-complement activity [1719]. Besides the immunomodulatory effects of Althaea officinalis, its extract can influence the proliferation of epithelial cells [20]. In vivo studies showed that this extract has protective effects against ultraviolet radiation, promotes wound healing, and suppresses the symptoms of latex allergic reactions [21-23]. Our results showed that the prescription of liposomal formulation of Althaea Officinalis flower extract is as efficient as hydrocortisone acetate ointment (1\%) or triamcinolone in the long-term in the controlling of symptoms of patients with atopic eczema.

In a study conducted by Naseri $\mathrm{V}$ et al. at [24], the effect of topical marshmallow in children with atopic dermatitis (AD) were examined. The efficacy of Althaea officinalis $1 \%$ ointment in a decrease of disease severity is more than Hydrocortisone 1\% in children with $\mathrm{AD}$. The results of our study was same as this study, but the main difference between these studies is the formulation of the drug. We formulated the liposomal form of herbal 
medicine that is the key point in topical drug dosage forms which increase the absorption, significantly.

In another recent study by Bonaterra GA et al. antiinflammatory and anti-oxidative effects of Phytohustil and Root Extract of Althaea officinalis (REAo) on Macrophages in vitro were studied. This in vitroinvestigation indicate protective, i.e., anti-oxidative and antiinflammatory effects of REAo and Phytohustil. These anti-inflammatory effects were similar or even better than diclofenac [12].

The main constitutes responsible for pain reduction and the inflammation suppression are known to be polyphenols, flavonoids, anthocyanins, dioxybutyric acid and terpenoids $[25,26]$. Allergic reactions may accurse as an adverse effects. We have not enough studies regarding to the bioavailability, although our product is topical, not oral.

As a limitation of the present study, we did not assess the pharmacokinetic and pharmacodynamic effects of Althaea officinalis. Therefore, and conclusion regarding understanding the mechanism of action of the drug cannot be derived. In future studies, authors recommend assessing the bioavailability of the liposomal formulation ofAlthaea Officinalis flower extract and the effect of its coadministration with corticosteroids on reducing topical corticosteroid dose.

\section{Conclusion}

Our results showed that although the standard treatment for atopic dermatitis lesions, which includes the use of steroid pads, can improve symptoms in a short time, but the liposomal formulation of Althaea Officinalis flower extract is clearly effective as steroids in a long time. Also, there was no side effect in the Althaea Officinalis flower group. Considering the potential side effects of steroids in a long-term use and similar efficacy of Althaea Officinalis flower extract, using this component may be accompanied by higher benefits in patients with atopic eczema.

\section{Acknowledgements}

The authors would like to express their gratitude to the staff of Qaem Educational Hospitals of Mashhad and all patients who participated in the current study.

\section{Authors' contributions}

Roshanak Salari: Conceptualization, Project administration, Supervision, Writing - Review \& Editing, Validation. Negar Khalighi: Writing - Original Drat. Farahzad Jabbari-Azad: Project administration, Supervision, Writing - Review \& Editing, Validation. Maral Barzegar-Amini: Software, Formal analysis, Writing - Review \& Editing, Visualization. Jalil Tavakkol-Afshari: Investigation, Resources, Writing - Review \& Editing. Pouran Layegh: Investigation. The author(s) read and approved the final manuscript.

\section{Funding}

Deputy for Research and Technology of Mashhad Medical University support their financial (code: 940692).

\section{Availability of data and materials}

The datasets used and/or analyzed during the current study are available from the corresponding author on reasonable request.

\section{Declarations}

Ethics approval and consent to participate

All procedures performed in studies involving human participants were following the ethical standards of the institutional and/or national research committee and with the 1964 Helsinki Declaration and its later amendments or comparable ethical standards. The protocol of the study was approved by the Ethics Committee of Mashhad University of Medical Sciences (IR.MUMS.fm.REC.1394.543).

\section{Consent for publication}

All Authors All authors are fully satisfied with the submission of the article for publication and the article was approved by them. Their personal information included:

Roshanak Salari: Department of Pharmaceutical Sciences in Persian Medicine, School of Persian and Complementary Medicine, Mashhad University of Medical Sciences, Mashhad, Iran. E-mail: salarir@mums.ac.ir, Tel: +98-5138829279

Negar Khalighi: Resident of Clinical Immunology and Allergy, Allergy Research Center, Mashhad University of Medical Sciences, Ghaem Hospital, Mashhad, Iran. Email: KhalighiN971@mums.ac.ir, Mobile: 00989153581171 Farahzad Jabbari-Azad: Professor of Clinical Immunology and Allergy, Allergy Research Center, Mashhad University of Medical Sciences, Ghaem Hospital, Mashhad, Iran. Email: jabbarif@mums.ac.ir, Mobile: 00989153167868 Maral Barzegar-Amini: Msc of Biochemistry, Allergy Research Center, Mashhad University of Medical Sciences, Ghaem Hospital, Mashhad, Iran. Email: aminim1@mums.ac.ir, Mobile: 00989155233290

Jalil Tavakkol-Afshari: Professor of Clinical Immunology, Immunology Research Center, Mashhad University of Medical Sciences, Mashhad, Iran. Email: Tavakolaj@mums.ac.ir, Mobile: 00989151134220

Pouran Layegh: Professor of Dermatology, Cutaneous Leishmaniasis Research Center, Mashhad University of Medical Sciences, Mashhad, Iran.

Email:layeghpo@mums.ac.irMobile: 00989155107107

\section{Competing interests}

There is no conflict of interest.

\section{Author details}

${ }^{1}$ Allergy Research Center, Mashhad University of Medical Sciences, Mashhad, Iran. ${ }^{2}$ Immunology Research Center, Mashhad University of Medical Sciences, Mashhad, Iran. ${ }^{3}$ Cutaneous Leishmaniasis Research Center, Mashhad University of Medical Sciences, Mashhad, Iran. ${ }^{4}$ Department of

Pharmaceutical Sciences in Persian Medicine, School of Persian and Complementary Medicine, Mashhad University of Medical Sciences, Mashhad, Iran.

Received: 16 November 2020 Accepted: 25 July 2021

Published online: 01 September 2021

\section{References}

1. Abuabara K, Magyari A, McCulloch CE, Linos E, Margolis DJ, Langan SM. Prevalence of atopic eczema among patients seen in primary care: Data from the health improvement network. Ann Intern Med. 2019;170(5):354-6.

2. Schonmann Y, Mansfield KE, Hayes JF, Abuabara K, Roberts A, Smeeth L, et al. Atopic eczema in adulthood and risk of depression and anxiety: a population-based cohort study. J Allergy Clin Immunol. 2020;8(1):248-57 e16.

3. Wernham A, Veitch D, Grindlay D, Rogers N, Harman K. What's new in atopic eczema? An analysis of systematic reviews published in 2017. Part 1: treatment and prevention. Clin Exp Dermatol. 2019;44(8):861-7.

4. Siriyong T, Phungtammasan S, Jansorn S, Chonsongkram N, Chanwanitsakul S, Subhadhirasakul S, et al. Traditional Thai herbal medicine as an alternative treatment for refractory chronic eczema. Explore. 2020;16(4):242-9.

5. Koo K, Nagayah R, Begum S, Mahmood TM, Shah NM. The use of complementary and alternative medicine in children with atopic eczema at a tertiary care centre in Malaysia. Complement Ther Med. 2020;49:102355. 
6. Al-Waili NS. Topical application of natural honey, beeswax and olive oil mixture for atopic dermatitis or psoriasis: partially controlled, single-blinded study. Complement Ther Med. 2003;11(4):226-34.

7. Al-Waili NS, Saloom KS, AI-Waili TN, Al-Waili AN. The safety and efficacy of a mixture of honey, olive oil, and beeswax for the management of hemorrhoids and anal fissure: a pilot study. ScientificWorldJournal. 2006;6: 1998-2005.

8. Dawid-Pać R. Medicinal plants used in treatment of inflammatory skin diseases. Postępy Dermatol Alergol. 2013;30(3):170.

9. Strzelecka H, Kowalski J. Encyclopedia of herbalism and herbal medicine. Warszawa: PWN; 2000.

10. Organization WH. WHO monographs on selected medicinal plants. Switzerland: World Health Organization; 1999.

11. Steinhoff B. E/S/C/O/P-the European scientific cooperative on phytotherapy. Phytomedicine. 2002;9(5):485.

12. Bonaterra GA, Bronischewski K, Hunold P, Schwarzbach H, Heinrich E-U, Fink $C$, et al. Anti-inflammatory and Anti-oxidative Effects of Phytohustil ${ }^{\oplus}$ and Root Extract of Althaea officinalis L. on Macrophages in vitro. Front Pharmacol. 2020;11:290.

13. Althaea officinalis. 2021. Available from: https://en.wikipedia.org/wiki/Altha ea_officinalis.

14. Benbassat N, Kostova B, Nikolova I, Rachev D. Development and evaluation of novel lozenges containing marshmallow root extract. Pak J Pharm Sci. 2013;26(6):1103-7.

15. Hon K, Leung T, Ng P, Lam M, Kam W, Wong K, et al. Efficacy and tolerability of a Chinese herbal medicine concoction for treatment of atopic dermatitis: a randomized, double-blind, placebo-controlled study. $\mathrm{Br}$ J Dermatol. 2007;157(2):357-63.

16. Thandar Y, Gray A, Botha J, Mosam A. Topical herbal medicines for atopic eczema: a systematic review of randomized controlled trials. Br J Dermatol. 2017;176(2):330-43.

17. Al-Snafi A. Immunological effects of medicinal plants: A review (part 2). Immun Endoc Metab Agents in Med Chem. 2016;16(2):100-21.

18. Yamada H, Nagai T, Cyong J-C, Otsuka Y, Tomoda M, Shimizu N, et al. Relationship between chemical structure and anti-complementary activity of plant polysaccharides. Carbohydr Res. 1985;144(1):101-11.

19. El WG, Ghanem EB, Chedid LA, Abdelnoor AM. The effects of Alcea rosea L., Malva sylvestris $L$. and Salvia libanotica $L$. water extracts on the production of anti-egg albumin antibodies, interleukin-4, gamma interferon and interleukin-12 in BALB/c mice. Phytother Res. 2008;22(12):1599-604.

20. Deters A, Zippel J, Hellenbrand N, Pappai D, Possemeyer C, Hensel A. Aqueous extracts and polysaccharides from Marshmallow roots (Althea officinalis $\mathrm{L}$.): Cellular internalisation and stimulation of cell physiology of human epithelial cells in vitro. J Ethnopharmacol. 2010;127(1):62-9.

21. Curnow A, Owen SJ. An evaluation of root phytochemicals derived from althea officinalis (Marshmallow) and astragalus membranaceus as potentia natural components of UV protecting dermatological formulations. Oxid Med Cell Longev. 2016:1:1-9.

22. Miroliaei M, Chelongar R, Aminjafari A, Talebi A, Ghiyas M. Histopathological evaluation of non-infectious skin deep wound healing activity of herbal extract. 2017;30(2):212-22.

23. Jafarimanesh $\mathrm{H}$. The effect of Alcea Althea on latex allergy among operating room staffs in Arak Hospitals, Iran. Complement Med J Faculty Nurs Midwifery. 2015;4(4):954-67.

24. Naseri V, Chavoshzadeh Z, Mizani A, Daneshfard B, Ghaffari F, AbbasMohammadi $\mathrm{M}$, et al. Effect of topical marshmallow (Althaea officinalis) on atopic dermatitis in children: A pilot double-blind active-controlled clinical trial of an in-silico-analyzed phytomedicine. Phytother Res. 2021;35(3):138998.

25. Miguel MG, Anthocyanins. Antioxidant and/or anti-inflammatory activities. J Appl Pharm Sci. 2011;1(6):7-15.

26. Muthiah N, Viswanathan S, Thirugnanasambntham P, Reddy M. AntiInflammatory Activity of Flavone and its Methoxy Derivatives-A Structure Activity Study. Indian J Pharm Sci. 1993:55:180.

\section{Publisher's Note}

Springer Nature remains neutral with regard to jurisdictional claims in published maps and institutional affiliations.

\section{Submit your manuscript to a SpringerOpen ${ }^{\circ}$ journal and benefit from:}

- Convenient online submission

- Rigorous peer review

- Open access: articles freely available online

High visibility within the field

- Retaining the copyright to your article

Submit your next manuscript at $\boldsymbol{\nabla}$ springeropen.com 